\title{
Philosophiques
}

\section{Remarques sur le livre de Jean-François Kervégan}

\section{Olivier Beaud}

Volume 39, numéro 2, automne 2012

URI : https://id.erudit.org/iderudit/1013699ar

DOI : https://doi.org/10.7202/1013699ar

Aller au sommaire du numéro

Éditeur(s)

Société de philosophie du Québec

ISSN

0316-2923 (imprimé)

1492-1391 (numérique)

Découvrir la revue

Citer ce document

Beaud, O. (2012). Remarques sur le livre de Jean-François Kervégan.

Philosophiques, 39(2), 463-467. https://doi.org/10.7202/1013699ar d'utilisation que vous pouvez consulter en ligne.

https://apropos.erudit.org/fr/usagers/politique-dutilisation/ 


\title{
Remarques sur le livre de Jean-François Kervégan
}

\author{
OLIVIER BEAUD \\ Université Paris II, Panthéon-Assas \\ olivier.beaud@u-paris2.fr
}

Christian Nadeau m'a invité à l'art délicat de la disputatio en me demandant de commenter le livre de Kervégan intitulé "Que faire de Schmitt? ». L'exercice s'avère en l'espèce un peu délicat dans la mesure où cet auteur est un ami - et il est toujours difficile de commenter le livre d'un ami car l'objectivité requise n'est pas toujours facile à atteindre - , mais aussi du fait que je partage l'essentiel de ses opinions ici exprimées. Le terme de "disputatio » ne convient donc pas tout à fait pour décrire le propos que je vais tenter de tenir pour honorer la promesse que j'ai faite de rendre compte de ce livre pour le public canadien.

Il me faut d'abord saluer le tour de force que constitue la rédaction de cet ouvrage qui constitue à la fois une sorte d'introduction à la pensée de Schmitt et une réflexion sur les apports de celle-ci à la théorie politique et juridique. Seule une connaissance intime et profonde de la pensée du juriste allemand pouvait autoriser l'auteur à tenter une telle synthèse. D'une part, elle est d'une remarquable clarté pédagogique, et à ce titre vaut comme une pénétrante introduction à l'endroit de ceux qui ne connaîtraient pas la pensée de Schmitt, d'autre part, elle est à l'intention des spécialistes une sorte de prise de position critique, soutenue par une érudition sans faille ${ }^{1}$, qui enrichit considérablement la littérature proliférante de la Schmitt-Forschung. Il n'est pas si courant de réussir à maintenir deux niveaux de lecture quand on est spécialiste d'un auteur ${ }^{2}$, de sorte qu'on peut soutenir que ce livre apparaît comme la meilleure synthèse existant en français sur l'œuvre du juriste allemand.

L'ouvrage ne se contente pas seulement de présenter les principaux thèmes de sa pensée, dans les cinq chapitres de la seconde partie (théologien du droit, normativisme, légitimité, politique et «unité du monde»), mais il explore aussi des questions centrales que l'actualité de sa pensée analysée tant à travers la résonance actuelle de ses concepts fondamentaux que des multiples réceptions d'un auteur qui est devenu «à la mode » ces dernières années, qu'on le regrette ou non, mais qui continue à être considéré comme

1. Il n'est pas inutile de souligner que, germaniste éminent, Kervégan cite tous les textes à partir de l'original allemand. Il faudrait signaler à l'auteur, si une seconde édition devait avoir lieu, que Gesetz und Urteil (I9I 2) n'était pas la thèse d'habilitation de Schmitt.

2. D'un certain point de vue, l'ouvrage peut se comparer avec la commode synthèse de R. Mehring, Carl Schmitt zur Einführung, mais seulement quant à la dimension pédagogique. Pour le reste, le livre de Kervégan est d'une exigence bien supérieure. 
un auteur suspect en raison de sa grave compromission avec les nazis. Bien que son auteur ne le dise pas explicitement, on devine vite que l'origine de ce livre résulte de la vive polémique lancée, en février 2007 , dans le journal Le Monde par Yves-Charles Zarka (philosophe et directeur de la revue Cités) qui avait identifié la pensée schmittienne comme une pensée nazie et avait exhorté les lecteurs du journal à prendre ses écrits comme des «documents» et non comme des œuvres. Il suffit, pour s'en convaincre, de rappeler que cette tribune, avait précisément pour titre "Que faire de Carl Schmitt? ». À l'époque, sans avoir pris parti dans la querelle, Kervégan avait participé avec d'autres connaisseurs de Schmitt à une mise au point distanciée dans la revue Le Débat. D’une certaine manière, ce livre est une tentative de mise au point, de la part d'un philosophe qui, connu pour sa science hégélienne, s'est aussi frotté à la pensée schmittienne tout au long de sa thèse d'État ${ }^{3}$.

En raison de ce passé pas si lointain, l’ouvrage commence donc à juste titre par l'interrogation brûlante: que faire d'un auteur aussi gravement compromis avec Hitler? - question qui n'est pas sans rappeler les interrogations suscitées par la même compromission commise par Heidegger. Peuton ou non faire abstraction d'un engagement idéologique aussi «lourd » ? À cet égard, il me semble que les opinions exprimées par Kervégan dans la première partie de son livre représentent la meilleure position qui vise à s'écarter autant de la thèse continuiste que de la thèse de la "parenthèse " (p. 35 ). Les partisans de la première, qui sont donc les anti-schmittiens virulents, estiment que l'aboutissement de l'œuvre weimarienne de Schmitt figure dans son engagement nazi qui fut particulièrement net entre I933 et I936, mais tout aussi net entre I936 et I942, tandis que les partisans de la seconde thèse, les apologètes de sa pensée, n'y voient qu'une malheureuse parenthèse, fruit d'un opportunisme de mauvais aloi. Pour sortir de ce qu'il considère comme une alternative infructueuse, Kervégan tente de distinguer radicalement l'œuvre de l'homme et nous invite à nous démarquer de la tentative visant «à interpréter l'œuvre entière à partir de l'engagement nazi de son auteur» (p. 5I). Une telle méthode contient aussi une prise de position sur un des débats les plus récurrents dans la Schmitt-Forschung qui consiste à se demander si cet auteur a une doctrine établie ou s'il n'a pas au contraire une pensée par trop mouvante qui épouserait - trop d'ailleurs - les circonstances de l'époque. Dans ce débat, la position de Kervégan est très nette: il considère que les "préoccupations théoriques" de Schmitt "sont d'une grande stabilité » (p. 5I). Et il identifie les deux grands foyers de sa pensée: "d'un côté, "la question du devenir de l'État et de ses prédicats classiques", et d'un autre côté, "le problème d'une éventuelle dilution de la dimension politique de la vie humaine”" (p. $5 \mathrm{I})$.

3. On peut d'ailleurs regretter que la grande presse, si prompte à traiter Schmitt comme un sujet de polémique, n'ait pas cru bon de recenser ce livre. 


\section{Remerques sur le livre deJeen-François Kenégan • 465}

Il n'y aurait pas grand intérêt à résumer le contenu de ce livre, que j'ai évoqué plus haut, sauf à dire que les thèmes retenus correspondent effectivement aux grands enjeux de la pensée de $\mathrm{Schmitt}^{4}$. De manière plus subjective, j'évoquerai ici le grand plaisir éprouvé à redécouvrir les grands thèmes de sa pensée et, en le lisant, à me remémorer les raisons pour lesquelles son œuvre m'avait interpellé lorsque je travaillais, dans ma thèse, sur le concept de souveraineté de l'État. Dans cette lecture, volontairement partielle du livre, j'ai retenu de celle-ci la mise en évidence par Kervégan de deux thèmes, selon moi essentiels. Le premier, c'est ce qu'il appelle très justement «le moment politique du droit» (p. I89) - ou encore "l'instauration politique $d u$ droit» (p. I90) — par lequel Schmitt entend, contre Kelsen et le positivisme, montrer que la Constitution ne peut pas être comprise si on entend la clore sur le seul droit positif, la question de "la légitimité de la légalité» surplombant toujours le droit constitutionnel. De ce point de vue, la fin du chapitre III sur le normativisme et le chapitre entier sur la légitimité (chap. V) me paraissent, pour tout juriste s'intéressant aux questions constitutionnelles, d'une importance majeure. Rétrospectivement, j’ai retrouvé presque intacte la profonde impression que m'avait laissé le traitement, fait par Schmitt, du pouvoir constituant dans la Verfassungslehre. Qu'on le veuille ou non, pour tous les juristes qui ne se satisfont pas de la littérature dominante sur le droit constitutionnel, lequel ne tend à devenir en France - plus tard d'ailleurs - que le positivisme du contentieux constitutionnel, la lecture de Schmitt s'avère un antidote efficace; le moindre paradoxe de ce livre tient donc à ce qu'un philosophe, Kervégan, l'indique à la corporation des juristes. Le second thème n'est autre que celui de l'État et de la question de son éventuel dépérissement. On a vu que la question de l'État était restée centrale dans la pensée schmittienne; elle est abordée ici longuement dans les deux derniers chapitres sur la politique (chap. VI) et sur l'unité du monde" (chap. VII). Ici se révèle un des grands apports de ce livre, qui est de reconsidérer grandement l'œuvre schmittienne d'après I945 (et un peu des années I940-I945 avec l'essai sur les "grands espaces»), laquelle est principalement consacrée au droit international public et dont la clé, selon Kervégan, est l'interrogation sur la péremption de l'État. Ce diagnostic, posé en I942, débouche sur la question de savoir par quoi va être remplacée la souveraineté des États, qui structurait l'ordre politique westphalien et que Schmitt réinterprète à sa façon comme le vieux ius publicum europaeum. Cela revient à se poser non seulement la question des nouvelles formes politiques, et ici l'empire apparaît comme le successeur paradoxal de la forme étatique, mais aussi celle de la survie du «politique» dans un monde déséta-

4. D'ailleurs, on dispose déjà de quelques recensions auxquelles on peut renvoyer le lecteur: ainsi la recension de Jacky Hummel (l'auteur d'un précieux Schmitt, chez Michalon, qui n'est curieusement pas cité par Kervégan, dont la bibliographie est pourtant impressionnante) dans Jus Politicum, n 99. 
tisé. Un des grands intérêts du livre ici est de montrer l'évolution de la pensée de Schmitt et aussi d'indiquer les multiples résonances de sa pensée dans le monde contemporain, comme l'illustrent la «guerre contre le terrorisme » et l'instrumentalisation des organisations internationales au profit des grandes puissances dominantes.

S'il fallait absolument pointer quelques désaccords ou réticences, je me bornerais à évoquer deux points seulement. Il me semble que Kervégan est un peu trop généreux dans sa façon de décrire les opinions de Schmitt sur la République de Weimar et sur la démocratie (notamment p. I 58 - I 59) tant il paraît, selon moi, difficile d'accepter la vision de la démocratie et de la «légitimité démocratique» proposée par celui-ci. La légitimité plébiscitaire telle qu'elle est invoquée dans la Théorie de la constitution s'éloigne considérablement du correctif subtil qu'avait en tête, me semble-t-il, Max Weber. C'est peut-être sur ce point qu'on découvre l'ambiguité de l'expression: "partir de Carl Schmitt» au premier sens du terme (p. 73) - et non pas au second qui est "prendre congé » de ses thèses - car souvent, en matière intellectuelle, c'est la manière de poser les problèmes qui prédétermine les réponses à la question posée. Or, de ce point de vue, il n'est pas du tout certain que la manière dont $S$ chmitt pose le problème de la démocratie soit la mieux adaptée pour répondre aux questions de notre temps.

L'autre interrogation porte sur un point essentiel qui est à la fois un point d'accord et de désaccord. Kervégan considère qu'il faut étudier Schmitt non pas comme un philosophe politique, ou un théologien politique, mais « comme un juriste» (p. 24, et aussi note 4 p. 280); il reprend d'ailleurs une affirmation de Schmitt lui-même: "J'ai toujours écrit et parlé en tant que juriste, et par conséquent seulement à des juristes et pour des juristes. » Bien que je partage cette prémisse, il m'apparaît que Kervégan ne respecte pas toujours ce précepte. Ainsi, quand il écrit que Schmitt est un «classique» dans la pensée politique (p. Io) ou «notre Machiavel» (p. 25I), il cesse de le considérer comme un juriste pour en parler essentiellement comme d'un théoricien du politique, tout comme d'ailleurs quand il explique la réception de Schmitt en France par le déclin du marxisme et la nécessité de trouver des auteurs critiquant le libéralisme (chap. II), car une telle explication ne rend pas compte de la réception, tardive, de sa pensée chez les juristes français (disons chez les juristes de droit public).

Poursuivons cette remarque par une interrogation qui se veut ouverte, et qui reste pour moi une énigme. La question est de savoir pourquoi Schmitt, juriste de formation, et professeur de droit, peut à la fois passionner les philosophes et exaspérer les juristes positivistes. D’un côté, il passionne Heidegger, il est lu par Walter Benjamin, Jünger, et une partie de sa notoriété lui vient des non-juristes. Mais d'un autre côté, chez les juristes, il est un marginal et il est resté un marginal. Les juristes de droit international public ne le citaient presque jamais, jusqu'à une date très récente, tant sa manière de pratiquer cette discipline leur paraissait aux antipodes de leur art. N'est-ce pas d'ail- 
leurs un brillant internationaliste, Joseph Kunz, un proche de Kelsen, qui déclare que "Schmitt n'est pas un juriste, mais un "politicien " (a politician) (cité ici par Kervégan, p. 223). D’autres juristes de droit public aussi réputés que Richard Thoma ou Rudolf Smend le tiendront toujours à distance ${ }^{5}$, de sorte que Schmitt cherchera en vain la reconnaissance de la part de la corporation du droit public, qui le trouvera toujours un peu suspect, même avant I933. On pourrait d'ailleurs ajouter le fait que Schmitt est un juriste de droit public, un Staatsrechtslehrer, qui examine prioritairement le rapport entre le pouvoir et le droit, mais dont la pensée semble avoir peu intéressé ses collègues privatistes de l'époque, tel un Karl Larenz, par exemple, qui semble pourtant avoir eu un destin assez similaire au $\operatorname{sien}^{6}$; de même, il est patent que, de nos jours, en France, les juristes de droit privé (les privatistes) et les internationalistes sont plus que rétifs à son endroit. Si le livre explique fort bien ce que signifie l'expression cryptée de «théologien du droit» (p. I I4) utilisée par Schmitt pour se définir, il ne s'interroge pas assez à mon avis sur la validité de cette assertion du juriste qui «parle en juriste et pour les autres juristes ». Mais Kervégan, qui est philosophe, peut très bien rétorquer que c'est justement une question à laquelle les juristes devraient eux-mêmes répondre... Il a fait tout autre chose, et fort bien, dans ce livre qui fera date dans les études schmittiennes.

5. Comme le prouve la publication récente de la correspondance entre Schmitt et Smend Auf der gefahrenvollen Straße des öffentlichen Rechts. Briefwechsel Carl Schmitt - Rudolf Smend 1921-1961, Duncker und Humblot Verlag, Berlin 2010 (hrsg Mehring).

6. Voir la présentation de l'homme et de son œuvre par son élève, Claus-Wilhelm Canaris, «Karl Larenz ", in Grundmann/Riesenhuber (Hrsg.), Zivilrechtslehrer des 20. Jabrhunderts in Berichten ibrer Schüler, Band 2, 2010, p. 263-308. 